\title{
Prevalence of intestinal parasitic infections among school children in capital areas of the Democratic Republic of São Tomé and Príncipe, West Africa.
}

\author{
Chien-Wei Liao ${ }^{1}$, Chung-Jung Fu ${ }^{2,3}$, Cheng-Yan $\mathrm{KaO}^{2}$, Yueh-Lun Lee ${ }^{4}$, Po-Ching Chen ${ }^{1}$, \\ Ting-Wu Chuang ${ }^{1}$, Toshio Naito ${ }^{5,6}$, Chia-Mei Chou ${ }^{1}$, Ying-Chie Huang ${ }^{1}$, Idalina Bonfim ${ }^{7}$, Chia-Kwung Fan ${ }^{1,8,9}$
}

1. Department of Molecular Parasitology and Tropical Diseases, School of Medicine, College of Medicine, Taipei Medical University, Taipei, Taiwan

2. Graduate Institute of Biomedical Electronics and Bioinformatics, National Taiwan University, Taipei, Taiwan

3. Department of International Medical Affairs, Taipei Medical University-Shuang-Ho Hospital, Taipei, Taiwan

4. Department of Microbiology and Immunology

5. Department of General Medicine

6. Department of Infection Control Science, Juntendo University School of Medicine, Japan

7. Instituto de Ciências da Saúde Dr Victor Sá Machado, Democratic Republic of Sao Tomes and Principe

8. Master Program in Global Health and Development, College of Public Health and Nutrition, Taipei Medical

University, Taipei, Taiwan

9. International Master/PhD Program in Medicine, College of Medicine, Taipei Medical University, Taipei, Taiwan

\begin{abstract}
Background: Although the Democratic Republic of Sao Tome and Principe (DRSTP) has undertaken school children-based deworming programs against intestinal parasitic infections (IPIs) using a single dose of mebendazole annually since 2005, it remains unclear as to the outcome to date. The present study intends to investigate the recent IPIs status among school children living in capital areas of the DRSTP.

Methods: A total of 252 school children (121 boys and 131 girls) of grades 4 and 5 from 4 primary schools located in the capital areas participated in the present study and their fresh fecal specimens were examined for the presence of any parasites using the merthiolate- iodine-formaldehyde concentration method as conducted.

Results: The overall prevalence of IPIs was $64.7 \%$ (163/252). No significant gender difference in prevalence between boys $(67.8 \%)$ and girls $(61.8 \%)$ was found $(p=0.3)$.

The majority of school children were infected with a single species of parasite (55.8\%). Altogether, 12 different intestinal parasite species were identified in DRSTP school children, of which 9 species were pathogenic and the remaining 3 were non-pathogenic. Conclusion: Improving the detection method, sanitation facilities and personal hygiene as well as utilizing combined drugs are all important measures to greatly reduce IPIs in DRSTP school children.

Keywords: Democratic Republic of Sao Tome and Principe, school children, intestinal parasitic infections

DOI: http://dx.doi.org/10.4314/ahs.v16i3.8

Cite as: Liao C-W, Fu C-J, Kao C-Y, Lee Y-L, Chen P-C, Chuang T-W, Naito T, Chou C-M, Huang Y-C, Bonfim I, Fan C-K. Prevalence of intestinal parasitic infections among school children in capital areas of the Democratic Republic of São Tomé and Principe, West Africa. Afri Health Sci 2016;16(3): 690-697. DOI:http:/ / dx.doi.org/10.4314/abs.v16i3.8
\end{abstract}

\section{Corresponding author:}

Chia-Kwung Fan,

Department of Molecular Parasitology

and Tropical Diseases, School of Medicine,

College of Medicine, Taipei Medical University,

Taipei, Taiwan

Tel.\& Fax.: +886-2-27395092

E-mail: tedfan@tmu.edu.tw

\section{Introduction}

Intestinal parasitic infections (IPIs) still constitute one of the major causes of public health problems in the world, particularly in developing countries. They are estimated to affect around 3.5 billion people globally, and 450 million are ill as a result of these infections ${ }^{1}$. The fecal-oral route is significant in the transmission of IPIs to humans via poor personal hygiene, environmental conditions such as contamination of soil and water sources by human feces, 
and poor sewage disposal such as the use of night soil as fertilizer; therefore IPIs occur wherever there is poverty $^{2}$. IPIs deprive the poorest of the poor of their health, contributing to economic instability and social marginalization. People of all ages are affected by IPIs; however, children are the worst affected ${ }^{3}$.

IPIs due to intestinal protozoa and helminths are responsible for some of the most devastating and prevalent diseases of humans, i.e., Ascaris lumbricoides, Trichuris trichiura and hookworm, collectively referred to as soiltransmitted helminths (STHs), and are the most common intestinal parasites ${ }^{4}$; while Giardia intestinalis and Entamoeba histolytica, which cause diarrhea are the most prevalent intestinal protozoan parasites worldwide with about 200 million people currently infected ${ }^{5-7}$. Another common intestinal protozoon is Blastocystis hominis ${ }^{8}$.

Epidemiological evidence worldwide shows that schoolaged children (SAC) are more likely to be exposed to IPIs, and they are also more likely to be more heavily infected than other age groups ${ }^{9}$. Since the worm burden is associated with the severity of morbidity, these SAC are likely to suffer most at an age when they are both growing and learning. Several studies indicated that STHs and/or some intestinal protozoan infections are highly associated with lower scores on tests of mental performance and educational achievement in SAC investigated in Africa and Central and South American countries ${ }^{10-12}$.

Although limited information is available regarding the IPIs status among inhabitants of the Democratic Republic of Sao Tome and Principe (DRSTP) ${ }^{13-17}$, like other developing countries, IPIs are supposedly a major health problem in the DRSTP due to tropical climatic and poor hygienic conditions. However, IPIs studies in SAC are quite rare; only two studies conducted in 1987 and 2005 indicated that the prevalence of $A$. lumbricoides, T. trichi- ura, and some intestinal protozoan infections were fairly high in SAC ${ }^{13-17}$. Largely from 2005 onwards, the DRSTP accepted the advice of non-governmental organizations (NGOs) to begin SAC-based deworming program using a single-dose mebendazole regimen annually.

Although several years have passed, the effectiveness of the deworming program of SAC remains unclear to date due to a lack of a systematic follow-up study.

We monitored the deworming effectiveness by investigation of the recent IPIs status among primary school children living in capital areas of the DRSTP. The study may help the DRSTP delineate effective measures against IPIs to improve the health status of DRSTP children.

\section{Materials and methods}

Geographic description, study population and subject selection

The DRSTP consists of the two islands of Sao Tome and Principe and a number of smaller islets in the Gulf of Guinea. Sao Tome lies approximately $290 \mathrm{~km}$ from Gabon on the West African coast, and the equator crosses its Southern tip. The climate is tropical with two rainy seasons. The total number of inhabitants in the DRSTP is estimated to be 160,000 , and the total number of inhabitants on Sao Tome Island is approximately 150,000. This study was carried out between $6^{\text {th }}$ and $24^{\text {th }}$ October 2010. School children of grades 4 and 5 (with a mean age \pm standard deviation (SD) of $9.8 \pm 1.3 \mathrm{yr}$ ) from 4 primary schools (SM, PT, PG and DJ) located in the capital areas (Figure 1) who had not yet been incorporated into the annually routine deworming program as well as these areas were reported as having poor sanitation, similar living condition, were endemic for parasitic infections, and had unsafe water supply were selected for enrollment in the present study according to suggestions of the Ministry of Health and Social Affairs. 
Figure 1

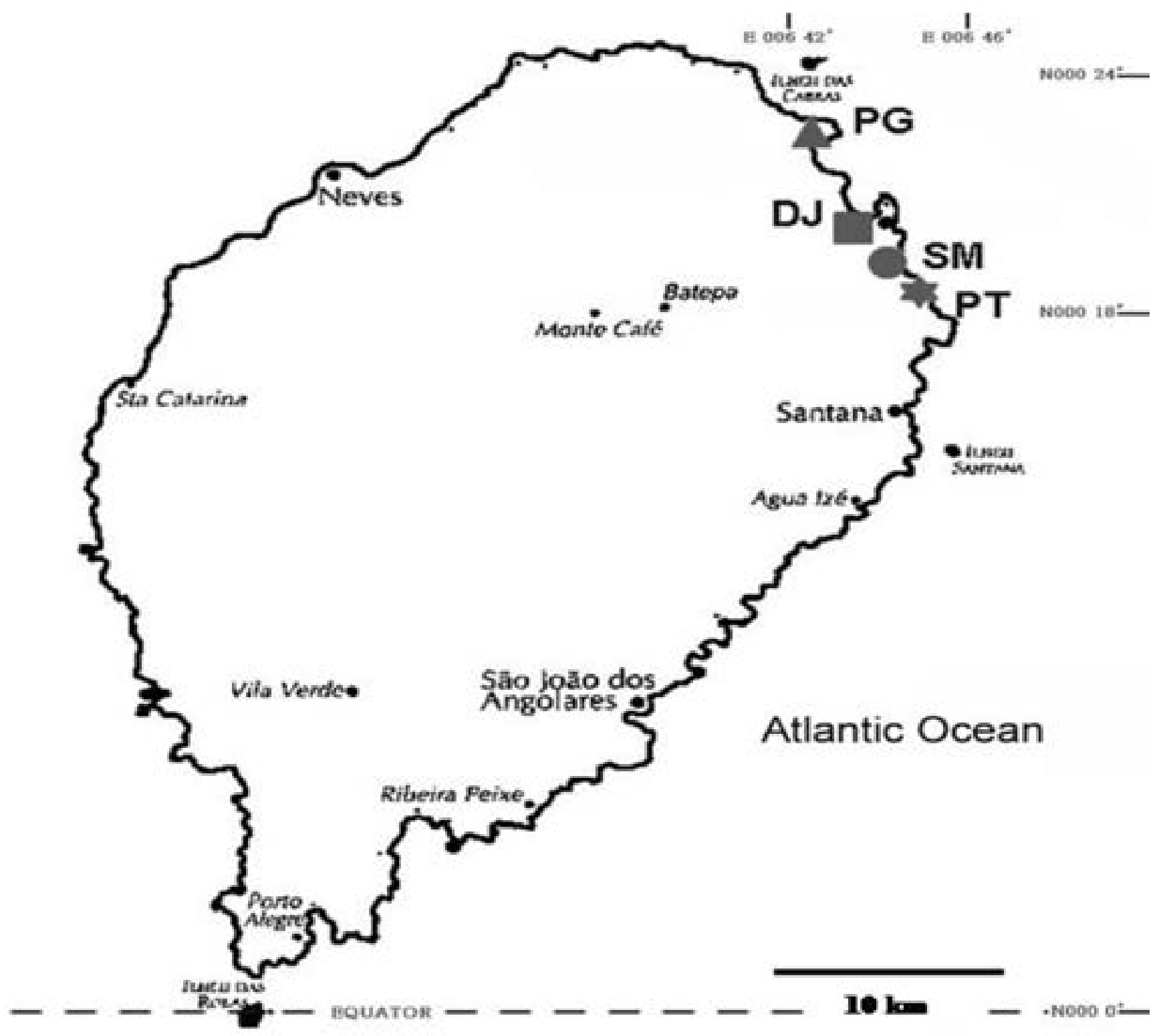

\section{Stool sample collection}

Prior to obtaining school children's fecal materials to examine them for the presence of any helminth ova, larva and/or protozoan cysts/trophozoite, we distributed stool containers (cat. no. ParaQ1, High Skill Inn., Chubei, Taiwan) along with a data sheet containing personal information, e.g., school, grade, name, gender and age, to the participating school children and asked them to bring the stool container back to us in the school after defecating the next morning. After we collected the stool containers from participating school children, individual fresh faecal specimens were processed within $4 \mathrm{~h}$ and examined for the presence of any parasites using the merthiolate-io- dine-formaldehyde (MIF) concentration method ${ }^{18}$. Overall, 252 stool samples were obtained, of which 121 stool samples were from boys and 131 stool samples were from girls. The mean ages were similar in both genders (boys: $9.8 \pm 1.4$ yr vs. girls: $9.7 \pm 1.3 \mathrm{yr}$ ).

\section{Parasitological examination}

To examine for parasites in the fecal materials, $5 \mathrm{ml}$ of a MIF solution containing $4.7 \mathrm{ml}$ of a merthiolate-formaldehyde solution and $0.3 \mathrm{ml}$ of an iodine solution (cat. no. ParaQ3, High Skill Inn.) were added to each stool container with a stool sample for at least $4 \mathrm{~h}$. The MIF- stool sample was then filtered through a layer of gauze in the 
bottom of the container, and the filtered solution in a collection tube (cat. no. ParaQ2, High Skill Inn.) was centrifuged at $1700 \mathrm{~g}$ for 2-3 min. The upper layer of clear fluid was discarded, and the residual pellet was examined for helminthic ova, larva and protozoan cysts/trophozoites under a microscope (Olympus BX41, Tokyo, Japan) ${ }^{18}$.

\section{Ethical approval}

Ethical approval for the study was obtained from the Ministry of Health and Social Affairs of the DRSTP. Written informed consent was obtained from parents, guardians or school representatives.

\section{Statistical analysis}

Statistical analyses were performed using SPSS software (SPSS, Chicago, IL, USA).
Crude odds ratios (ORs) with their 95\% confidence intervals $(\mathrm{CI})$ were estimated and $\mathrm{p}$ values of $<0.05$ were considered statistically significant.

\section{Results}

A total of 163 of the 252 school children were found to have IPIs, for an overall prevalence of $64.7 \%$ (Table 1). In addition, a significantly higher infection rate of pathogenic parasite $(49.2 \%)$ than non-pathogenic parasite $(15.5 \%)$ was found in primary school children (ORs $=5.3,95 \% \mathrm{CI}=3.5-8.1, \mathrm{p}<0.001)($ Table 1$)$.

Altogether, 12 different intestinal parasite species were identified in these DRSTP school children, of which 9 species are pathogenic parasites, namely $A$. lumbricoides, $T$. trichiura, Strongyloides stercoralis, hookworm, Schistosoma intercalatum, Hymenolepis nana, Entamoeba histolytica/E. dispar, G. intestinalis, and B. hominis.

Table 1 Prevalence, infection status and crude odds ratios (ORs) with $95 \%$ confidence intervals (CI) of intestinal parasitic infections among primary school children in capital areas of the Democratic Republic of São Tomé and Príncipe, West Africa

\begin{tabular}{|c|c|c|c|c|c|c|c|c|c|c|}
\hline \multirow[t]{2}{*}{ Subjects } & \multirow{2}{*}{$\begin{array}{l}\text { Age, } \\
\text { mean } \pm \\
\mathrm{SD} \\
\text { (in } \\
\text { year) }\end{array}$} & \multirow{2}{*}{$\begin{array}{l}\text { No. } \\
\text { examined }\end{array}$} & \multirow{2}{*}{$\begin{array}{l}\text { No. } \\
\text { positive } \\
(\%)\end{array}$} & \multicolumn{6}{|c|}{${ }^{\S}$ Pathogenic parasite (no., \%) } & \multirow{2}{*}{$\begin{array}{l}{ }^{*} \text { Non- } \\
\text { pathogeni } \\
\text { c parasites } \\
(\text { no., \%) }\end{array}$} \\
\hline & & & & "STHs & ${ }^{\top} \mathrm{OHs}$ & STHPP & "OHPP & ${ }^{\#} \mathrm{PP}$ & subtotal & \\
\hline Total & $\begin{array}{ll}9.8 & \pm \\
1.3 & \end{array}$ & 252 & $\begin{array}{l}163 \\
(64.7)\end{array}$ & $\begin{array}{l}28 \\
(11.1)\end{array}$ & $\begin{array}{l}8 \\
(3.2)\end{array}$ & $13(5.2)$ & $3(1.2)$ & $\begin{array}{l}72 \\
(28.6)\end{array}$ & $\begin{array}{l}124 \\
(49.2)^{\mathrm{c}}\end{array}$ & $39(15.5)^{\mathrm{C}}$ \\
\hline Boys & $\begin{array}{l}9.8 \\
1.4\end{array}$ & 121 & $\begin{array}{l}82 \\
(67.8)^{a}\end{array}$ & $\begin{array}{l}15 \\
(12.4)\end{array}$ & $\begin{array}{l}2 \\
(1.7)\end{array}$ & $6(5.0)$ & $1(0.8)$ & $\begin{array}{l}36 \\
(29.8)\end{array}$ & $\begin{array}{l}60 \\
(49.6)^{b}\end{array}$ & $22(18.2)^{\mathrm{d}}$ \\
\hline Girls & $\begin{array}{ll}9.7 & \pm \\
1.3 & \end{array}$ & 131 & $\begin{array}{l}81 \\
(61.8)^{\mathrm{a}}\end{array}$ & $\begin{array}{l}13 \\
(9.9)\end{array}$ & $\begin{array}{l}6 \\
(4.6)\end{array}$ & $7(5.3)$ & $2(1.5)$ & $\begin{array}{l}36 \\
(27.5)\end{array}$ & $\begin{array}{l}64 \\
(48.9)^{b}\end{array}$ & $17(13.0)^{\mathrm{d}}$ \\
\hline
\end{tabular}

Table 1 (cont) Prevalence, infection status and crude odds ratios (ORs) with 95\% confidence intervals (CI) of intestinal parasitic infections among primary school children in capital areas of the Democratic Republic of São Tomé and Príncipe, West Africa

\begin{tabular}{|l|l|l|l|l|l|}
\hline Subjects & $\begin{array}{l}\text { Age, mean } \pm \\
\text { SD } \\
\text { (in year) }\end{array}$ & $\begin{array}{l}\text { No. } \\
\text { examined }\end{array}$ & \multicolumn{2}{l|}{ Infection status (no., \%) } \\
\cline { 3 - 6 } & & & Single & Dual & Multiple \\
\hline Total & $9.8 \pm 1.3$ & 252 & $91(55.8)^{\mathrm{e}, \mathrm{f}}$ & $39(23.9)^{\mathrm{e}, \mathrm{g}}$ & $33(20.3)^{\mathrm{f}, \mathrm{g}}$ \\
\hline Boys & $9.8 \pm 1.4$ & 121 & $51(42.2)$ & $16(13.2)$ & $15(12.4)$ \\
\hline Girls & $9.7 \pm 1.3$ & 131 & $40(30.5)$ & $23(17.6)$ & $18(13.7)$ \\
\hline
\end{tabular}

${ }^{\S}$ Pathogenic parasite: ${ }^{*}$ STHs, soil-transmitted helminths including Ascaris lumbricoides, Trichuris trichiura, and hookworm; ${ }^{\dagger}$ OHs, other helminths includingStrongyloides stercoralis, Schistosoma intercalatum and Hymenolepis

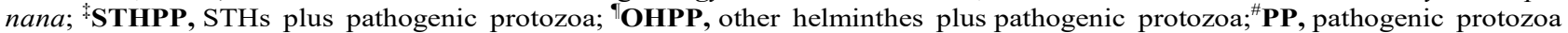
including Giardia intestinalis, Entamoeba histolytica/E. dispar; and Blastocystis hominis; ${ }^{*}$ Non- pathogenic parasites including Entamoeba coli,Endolimax nana, and Chilomastix mesnili.

${ }^{\mathrm{a}} \mathrm{ORs}=1.3,95 \% \mathrm{CI}=0.8-2.2, p=0.3 ;{ }^{\mathrm{b}} \mathrm{ORs}=1.0,95 \% \mathrm{CI}=0.6-1.7, p=0.9 ;{ }^{\mathrm{c}} \mathrm{ORs}=5.3,95 \% \mathrm{CI}=3.5-8.1, p<0.001 ;{ }^{\mathrm{d}} \mathrm{ORs}=$ $1.5,95 \% \mathrm{CI}=0.8-3.0, p=0.3 ;{ }^{\mathrm{e}} \mathrm{ORs}=3.1,95 \% \mathrm{CI}=2.0-4.7, p<0.001 ;{ }^{\mathrm{f}} \mathrm{ORs}=3.8 ; 95 \% \mathrm{CI}=2.4-5.9, p<0.001 ;{ }^{\mathrm{g}} \mathrm{ORs}=1.2$; $95 \% \mathrm{CI}=0.7-2.0 ; p=0.5$ 
The remaining 3 are non-pathogenic protozoa, namely Ent. coli, Endolimax nana and Chilomastix mesnili (Table 1).

Among pathogenic parasite, the protozoan (28.6\%, $72 / 252)$ and STHs $(11.1 \%, 28 / 252)$ infections were the most seen in primary school children (Table 1). No significant gender difference in the prevalence between boys $(67.8 \%, 82 / 121)$ and girls $(61.8 \%, 81 / 131)$ was found $(\mathrm{ORs}=1.3 ; 95 \% \mathrm{CI}=0.8-2.2 ; \mathrm{p}=0.3)$ (Table 1). Neither the prevalence of pathogenic parasites nor non-pathogenic parasites showed gender difference between boys and girls $(\mathrm{ORs}=1.0,95 \% \mathrm{CI}=0.6-1.7, \mathrm{p}=0.9 ; \mathrm{ORs}=$ 1.5; 95\% CI $=0.8-3.0 ; \mathrm{p}=0.3$ ) (Table 1$)$.

Regarding the number of IPIs in school children, the majority of school children were infected with a single species of parasite $(55.8 \%, 91 / 163)$ and that it was significantly higher than that of dual $(23.9 \%, 39 / 163)$ and multiple infections $(20.3 \%, 33 / 163)(\mathrm{ORs}=3.1,95 \% \mathrm{CI}$ $=2.0-4.7, \mathrm{p}<0.001 ; \mathrm{ORs}=3.8,95 \% \mathrm{CI}=2.4-5.9, \mathrm{p}<$ 0.001) (Table 1).

\section{Discussion}

IPIs remain a worldwide problem with an estimated 400 million school children worst affected. In sub-Saharan Africa, IPIs are a major health concern, because factors that predispose humans to infections, such as poverty, poor sanitation, ignorance, and malnutrition, prevail ${ }^{19}$. Although IPIs rarely directly cause death, they are associated with poor growth in children, vitamin deficiencies, irondeficiency anemia, and poor educational performance ${ }^{20}$; however, in the DRSTP, the severity and extent of the IPIs problem in inhabitants including children have been long neglected for a long time.

In 2001, during the 54th World Health Assembly, a resolution was passed that set a global target of scaling up intervention to achieve "regular treatment of at least $75 \%$ of SAC at risk of morbidity by STHs infections by $2010 " 21$. In order to achieve this target, the WHO recommends deworming all pre-school children and SAC with broad-spectrum antihelminthic drugs e.g., albendazole or mebendazole at regular intervals ${ }^{1}$. Recently, the DRSTP government was made aware of the severity and impact of IPIs on health and cognitive performance of SAC due to several investigations, which indicated a high prevalence of IPIs among $\mathrm{SAC}^{13,17}$.
In fact, even though deworming projects have been executed annually during 2006-2010, it is still difficult to obtain the data of IPIs prevalence due to no corresponding units followed up the outcome. However, informal data from some health officials was told that the IPIs prevalence after deworming for SAC ranged 50\% to 80\% around (personal communication). According to a formal report indicating that the prevalence of IPIs among SAC in rural areas of Lobata Province in DRSTP conducted in 2009 was high, reaching $52.2 \%(70 / 134)^{22}$.

The present study also found the high prevalence (64.7\%) of IPIs in SAC in capital areas. Both studies revealed that the deworming effect of using an annual single dose of mebendazole in DRSTP schoolchildren did not seem ideal due to the remained high prevalence of IPIs as found in different areas in $2009^{22}$ and 2010 (present study), respectively. As compared to other developing countries, present figure $(64.7 \%)$ was lower than that reported in Angola $(80 \%, 633 / 791)^{23}$; however it was significantly higher than that seen in Turkey $(31.8 \%, 145 / 456)$ and Palestine $(22.2 \%, 163 / 735)^{2,24}$; but a similar prevalence was seen in school children of Pakistan $(52.8 \%, 115 / 218)$ and Caribbean Islands of Saint Lucia $(61.6 \%, 341 / 554)^{3,25}$.

Although STHs and pathogenic intestinal protozoan infections constituted the largest proportion of IPIs among DRSTP school children, STHs prevalence $(11.1 \%)$ were greatly decreased as compared to those $(62.0 \%$ and $32.8 \%$ in previous studies conducted in $2005^{17}$ and $2009^{22}$, respectively, reflecting that the deworming program against STHs using a single dose of mebendazole annually seemed valid in DRSTP. However, several reports indicated that although mebendazole showed good therapeutic profiles against $A$. lumbricoides, it failed to effectively clear hookworm and T. trichiura infections ${ }^{26}$.Our recent study also found SAC orally given a single dosage of albendazole showed superior efficacy than mebendazole in reduction rate of STHs infection (albendazole : reduced from $43 \%$ to $8 \%$ vs. mebendazole : reduced from $70 \%$ to $43 \%$ ) (Fan et al., unpublished data). It was therefore suggested that using albendazole or a 6-month combination of mebendazole or albendazole with another drug, e.g., ivermectin, in DRSTP school children might achieve better outcomes against STHs and S. stercoralis infection. A similar finding was also seen in Vietnam ${ }^{27}$. 
They undertook a deworming program for Vietnamese school children using a single dose of oral mebendazole, but they found that it had low efficacy against hookworm infection compared to triple mebendazole or single albendazole in randomized controlled trials; thus they recommended using albendazole instead of mebendazole in deworming programs for Vietnamese $\mathrm{SAC}^{27}$. Knopp et al. (2010) suggested that either albendazole or mebendazole combined with ivermectin may improve therapeutic outcomes against T. trichiura and may be considered for use in STHs and S. stercoralis control programs ${ }^{28}$.

Intestinal pathogenic protozoa seem to raise much less interest than do STHs in most of African countries. In fact, intestinal pathogenic protozoan infections, e.g., Ent. bistolytica, G. intestinalis, and B. hominis, are more prevalent compared to helminthic infections as seen in DRSTP school children, and those pathogenic protozoa were commonly found together as well as non-pathogenic protozoa Ent. coli, End. nana, and C. mesnili which are all transmitted by the fecal-oral route. More importantly, polyparasitism or co-infection with either multiple intestinal helminths and/or protozoa in DRSTP school children is very common, and this phenomenon has already been reported in previous studies conducted in DRSTP school children in 1998, 2005, or 2009 and elsewhere in the other developing world ${ }^{13,17,20,22}$, indicating that most school children lack home sanitation (piped water and latrines) and thus had greater opportunities to contact contaminated soil and infected water when taking care of body hygiene and domestic activities ${ }^{17,22}$.

Intestinal amoebiasis, giardiasis, and blastocystiasis are very important parasitic diseases responsible for many deaths worldwide after malaria and schistosomiasis and most of them are frequent causes of diarrhea in preschool and SAC that can lead to malnutrition which can result in stunted growth, reduced physical fitness, impaired cognitive development and memory, and reduced school attendance ${ }^{9-12,20,29}$.

Therefore, infections by intestinal helminths, protozoa, or polyparasitism appear to be an indicator of a lack of access to clean water and improved sanitation facilities, and inadequate personal hygiene $e^{9,12,20}$. Improving environmental sanitation to prevent intestinal pathogenic protozoan and helminthic infections as well as properly treating infected school children by considering adding praziquantel against $S$. intercalatum and metronidazole against intestinal protozoon to mebendazole-or albendazole-alone regular deworming regimens to DRSTP SAC appear to be urgently needed.

Diagnostics for IPIs in medical laboratories of two major hospitals, e.g., the Central Hospital and Clinic Center, seemed problematic; both used an insensitive direct smear method. Although direct smear method can detect small-sized helminthic ova and protozoan cysts/ trophozoites, its disadvantage of this method cannot sometimes detect parasites in light infection. In fact, detection of parasites using this technique is based on high/low parasites in fecal samples and personal experience. Then, it led to low/no detection rates of IPIs in DRSTP.

So, we used MIF centrifugation technique better able to detect all species of various intestinal helminthic and protozoan parasites in fecal specimen, which is superior to simple smear method and good for low infection ${ }^{18,30,31}$. Because MIF can be standardized as one of basic parasitological protocols wherever IPIs are common, not only in DRSTP. Therefore, we strongly recommend that MIF be used as the detection system for IPIs in DRSTP hospitals instead of the direct smear method to greatly improve the detection rate of IPIs. Consequently, this will benefit the DRSTP in developing strategies for determining which anti-parasite drugs can efficiently be used for deworming regimens.

Taken together, SAC are the predominate targets for treatment, and school-based treatment delivery programs offer major cost advantages because of the use of the existing school infrastructure and the fact that schoolchildren are accessible through schools. Improving the detection method of IPIs, utilizing albendazole combined with other drugs, e.g., ivermectin, praziquantel, or metronidazole, in the annual deworming regimen against trematodes (e.g., $S$. intercalatum), cestodes (e.g., $H$. nana) and protozoa (e.g., Ent. histolytica and G. intestinalis) and improving sanitation facilities and personal hygiene through educational approach are all important measures to greatly reduce IPIs in DRSTP school children.

\section{Acknowledgements}

The authors are grateful to the Ministry of Health and Social Affairs of the Democratic Republic of Sao Tome 
and Principe. The authors also thank the Embassy of the Republic of China (Taiwan) in Democratic Republic of Sao Tome and Principe, the International Cooperation and Development Fund, Taiwan, and the Taiwanese Ministry of Foreign Affairs as well as Taipei Medical University (TMU98AE1-B19) for their support of this investigation.

\section{Conflict of interest}

All authors declare that they have no competing financial or any other interest in relation to their work.

\section{References}

1. Hotez PJ, Fenwick A, Savioli L and Molyneux DH. 2009. Rescuing the bottom billion through control of neglected tropical diseases. Lancet 2009; 373: 1570-1575.

2. Ojha SC, Jaide C, Jinawath N, Rotjanapan P and Baral P. Geohelminths: public health significance. Journal of Infection in Developing Countries 2014; 8: 5-16 PubMed.

3. Mehraj V, Hatcher J, Akhtar S, Rafique G and Beg MA. Prevalence and factors associated with intestinal parasitic infection among children in an urban slum of Karachi. PLoS One 2008; 3: e3680.

4. Bethony J, Brooker S, Albonico M, Geiger SM, Loukas A, Diemert D, H, et al. Soil- transmitted helminth infections: ascariasis, trichuriasis, and hookworm. Lancet 2006; 367: 1521-32.

5. Minenoa T and Avery MA. Giardiasis: recent progress in chemotherapy and drug development. Current Pharmaceutical Design 2003; 9: 841-55.

6. Stauffer W and Ravdin JI. Entamoeba histolytica: an update. Current Opinion in Infectious Diseases 2003; 16: 47985.

7. Ouattara M, N'Guessan NA, Yapi A and N'Goran EK. Prevalence and spatial distribution of Entamoeba histolytica/dispar and Giardia lamblia among schoolchildren in Agboville area (Cote d'Ivoire). PLoS Neglected Tropical Diseases 2010; 4: e574.

8. Karanis P, Kourenti C and Smith H. Waterborne transmission of protozoan parasites: a worldwide review of outbreaks and lessons learnt. Journal of Water and Health 2007; 5: 1-38.

9. Hotez PJ and Kamath A. Neglected tropical diseases in sub-saharan Africa: review of their prevalence, distribution, and disease burden. PLoS Neglected Tropical Diseases 2009; 3: e412.

10. Nokes C, Cooper ES, Robinson BA and Bundy DA.
Geohelminth infection and academic assessment in Jamaican children. Transaction of the Royal Society of Tropical Medicine and Hygiene 1991; 85: 272-73.

11. Boivin MJ, Giordani B, Ndanga K, Maky MM, Manzeki KM, Ngunu N, et al. Effects of treatment for intestinal parasites and malaria on the cognitive abilities of schoolchildren in Zaire, Africa. Health Psychology 1993; 12 : 220-6.

12. Berkman DS, Lescano AG, Gilman RH, Lopez SL and Black MM. Effects of stunting, diarrhoeal disease, and parasitic infection during infancy on cognition in late childhood: a follow-up study. Lancet 2002; 359: 564-71.

13. Pampiglione S, Visconti S, Visconti S and Pezzino G. Human intestinal parasites in Subsaharan Africa. II. Sao Tome and Principe. Parassitologia 1987; 29: 15-25.

14. Almeda J, Corachan M, Sousa A, Ascaso C, Carvalho JM, Rollinson D andSouthgate VR. Schistosomiasis in the Republic of Sao Tome and Principe: human studies. Transaction of the Royal Society of Tropical Medicine and Hygiene 1994; 88: 406-9.

15. Southgate VR, Rollinson D, Kaukas A, Almeda J, Sousa AM, Castro F,Soares E and Corachan M. Schistosomiasis in the Republic of Sao Tome and Principe: characterization of Schistosoma intercalatum. Transaction of the Royal Society of Tropical Medicine and Hygiene 1994; 88: 479-86.

16. Ripert C, Neves I, Appriou M, Tribouley J, TribouleyDuret J, Haumont G,Guy M and Trouvé B. Epidemiology of certain endemic parasitic diseases in the town of Guadalupe (Republic of Sao Tome and Principe) I. Schistosomiasisintercalatum and intestinal worms. Bulletin de la Société de Pathologie Exotique 1996; 89: 252-8.

17. Belo S, Rompao H, Goncalves L and Gracio MA. Prevalence, behavioural and social factors associated with Schistosoma intercalatum and geohelminth infections in Sao Tome and Principe. Parassitologia 2005; 47: 227-31.

18. Hsieh MH, Lin WY, Dai CY, Huang JF, Huang CK, Chien HH, Wang CL,Chung WL, Wu JR, Chen ER, Ho $\mathrm{CK}$ and $\mathrm{Yu}$ ML. Intestinal parasitic infection detected by stool examination in foreign laborers in Kaohsiung. Kaohsiung Journal of Medical Sciences 2010; 26:136-143.

19. Stephenson LS, Latham MC and Ottesen EA. Malnutrition and parasitic helminth infections. Parasitology 2000; 121: S23-S38.

20. Ngui R, Lim YAL, Chong KL, Sek C and Jaffar S.Association between anemia, iron deficiency anemia, neglected parasitic infections and socioeconomic factors 
in rural children of west Malaysia. PLoS Neglected Tropical Diseases 2012; 6: e1550.

21. World Health Assembly: Schistosomiasis and soil. transmitted helminth infections. Geneva: WHA; 2001. 22. Lobo ML, Augusto J, Antunes F, Ceita J, Xiao L, Codices V, Matos O.Cryptosporidium spp., Giardia duodenalis, Enterocytozoon bieneusi and other intestinal parasites in youngchildren in Lobata province, Democratic Republic of São Tomé and Principe. PLOS ONE 2014; 9: e97708.

23. Tomlinson M, Adams V, Chopra M, Jooste P, Strydom $\mathrm{E}$ and Dhansay A. Survey of iodine deficiency and intestinal parasitic infections in school-going children: Bie Province, Angola. Public Health Nutrition 2010; 13: 1314-8. 24. Hussein AS. Prevalence of intestinal parasites among school children in northern districts of West Bank-Palestine. Tropical Medicine and International Health 2011; 16: 240-244.

25. Kurup R and Hunjan GS. Epidemiology and control of Schistosomiasis and other intestinal parasitic infections among school children in three rural villages of southSaint Lucia. Journal of Vector Borne Diseases 2010; 47: 228-34.

26. Keiser J and Utzinger J. Efficacy of current drugs against soil-transmitted helminth infections: systematic review and meta-analysis. Journal of the American Medical Association 2008; 299: 1937-48.

27. Flohr C, Tuyen LN, Lewis S, Minh TT, Campbell J, Britton J, Williams H,Hien TT, Farrar J and Quinnell RJ. Low efficacy of mebendazole against hookworm in Vietnam: two randomized controlled trials. American Journal of Tropical Medicine and Hygiene 2007; 76: 732-6.

28. Knopp S, Mohammed KA, Speich B, Hattendorf J, Khamis IS, Khamis AN,Stothard JR, Rollinson D, Marti $\mathrm{H}$ and Utzinger J. Albendazole and mebendazole administered alone or in combination with ivermectin against Trichuris trichiura: a randomized controlled trial. Clinical Infectious Diseases 2010; 51: 1420-8.

29. Lobato L, Miranda A, Faria IM, Bethony JM and Gazzinelli MF.

30. Bhandari B, Gupta AP and Maheshwari CP. Merthiolate iodine formaldehyde test for detection of intestinal parasites. Indian Pediatrics 1984; 21: 803-6.

31. Duran C, Hidalgo G, Aguilera W, Rodriguez-Morales AJ, Albano C, Cortez J, Jiménez S, Díaz M and Incani RN.Giardia lamblia infection is associated with lower body mass index values. Journal of Infection in Developing Countries 2010; 4: 417-418. 